\title{
Thyroid Hormones within the Normal Range and Cardiac Function in the General Population: The EPIPorto Study
}

\author{
João Sérgio Neves ${ }^{a, b}$ Ricardo Fontes-Carvalho ${ }^{a, c}$ Marta Borges-Canha ${ }^{a, b}$ \\ Ana Rita Leite $^{a}$ Sandra Martins ${ }^{d, f}$ Ana Oliveira ${ }^{b}$ João Tiago Guimarães ${ }^{d-f}$

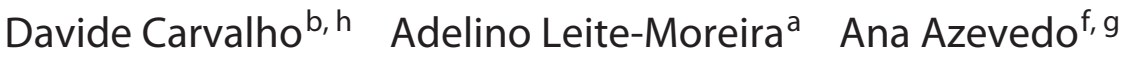 \\ a Unidade de Investigação Cardiovascular, Departamento de Cirurgia e Fisiologia, Faculdade de Medicina da \\ Universidade do Porto, Porto, Portugal; ${ }^{b}$ Department of Endocrinology, Diabetes and Metabolism, Centro \\ Hospitalar Universitário de São João, Porto, Portugal; ' Department of Cardiology, Centro Hospitalar Gaia/Espinho, \\ Vila Nova de Gaia, Portugal; ' d Department of Clinical Pathology, Centro Hospitalar Universitário de São João, \\ Porto, Portugal; ${ }^{e}$ Department of Biomedicine, Faculdade de Medicina da Universidade do Porto, Porto, Portugal; \\ ${ }^{f}$ EPIUnit - Instituto de Saúde Pública, Universidade do Porto, Porto, Portugal; ${ }^{9}$ Departamento de Ciências da \\ Saúde Pública e Forenses e Educação Médica, Faculdade de Medicina da Universidade do Porto, Porto, Portugal; \\ h Instituto de Investigação e Inovação em Saúde (i3S), Universidade do Porto, Porto, Portugal
}

\section{Keywords}

Thyroid hormones · Cardiac function - Cardiac structure .

Thyroid stimulating hormone · Free T4 · Free T3

\begin{abstract}
Background: Hypothyroidism and hyperthyroidism are associated with marked changes in cardiac structure and function. However, the association of thyroid function within the normal range with cardiac structure and function in the general population remains uncertain. Methods: Eight hundred thirty-five subjects aged $\geq 45$ years from the EPIPorto cohort (evaluation between 2006 and 2008) were cross-sectionally analyzed. We excluded participants with TSH, free T4 (FT4), or free T3 (FT3) outside of the reference range or with self-reported cardiovascular or thyroid disease. Cardiac structure and function were evaluated by echocardiogra-
\end{abstract}

phy. We used linear regression models unadjusted and adjusted for sex and age (model 1), and sex, age, BMI, diabetes, hypertension, and smoking (model 2). Nonlinear associations were assessed using restricted cubic splines. Results: The mean age was 61.5 years (SD 10.5); $61.1 \%$ of the patients were women. In the adjusted model 2 , heart rate was positively associated with FT3; diastolic blood pressure was positively associated with TSH; LV end-diastolic and end-systolic volumes were inversely associated with TSH, and ejection fraction was nonlinearly associated with FT3, with higher ejection fractions near the limits of the reference range. Left ventricle (LV) posterior wall thickness was nonlinearly associated with FT4 in the adjusted model 1, with a greater thickness near the limits of the reference range. Regarding diastolic function, no significant associations were observed in adjusted models. Conclusions: Thyroid function within the reference range was associated with heart rate, blood pres- 
sure, cardiac structure, and function. Increasing thyroid function (lower TSH, higher FT4, or higher FT3) was associated with a higher heart rate, a lower diastolic blood pressure, and larger LV volumes. LV wall thickness and ejection fraction had a U-shaped association with thyroid hormones.

(C) 2020 European Thyroid Association Published by S. Karger AG, Basel

\section{Introduction}

Cardiovascular disease is the leading cause of death worldwide. Traditional risk factors, including diabetes, dyslipidemia, and hypertension, contribute significantly to the development of cardiovascular disease [1]. Intensive treatment of these risk factors is associated with an important reduction of the risk of cardiovascular events [2-4]. However, even after correcting these risk factors, many patients still have a significant residual risk, highlighting the importance of understanding other mechanisms of cardiovascular dysfunction $[5,6]$.

Thyroid hormones play a critical role in cardiovascular system development and homeostasis [7]. Both overt hypothyroidism and hyperthyroidism contribute to a high risk of atherosclerotic disease and heart failure [810]. Even subclinical thyroid dysfunction is known to increase the cardiovascular risk, with both subclinical hypothyroidism and hyperthyroidism being associated with heart failure and atherosclerotic disease [11-15]. Furthermore, subclinical hyperthyroidism has been associated with an increased incidence of cardiac arrhythmias, particularly atrial fibrillation $[15,16]$, and subclinical hypothyroidism has been linked to an increased risk of hypertension $[17,18]$.

While the effects of overt and subclinical thyroid dysfunction on cardiac function have been well characterized $[19,20]$, the association of thyroid hormone levels within the reference range with cardiac function remains uncertain. Therefore, we aimed to evaluate the association between thyroid function within the reference range and cardiac function in the general population.

\section{Materials and Methods}

\section{Study Design and Participants}

The EPIPorto study is a population-based cohort study [21], ongoing for $>20$ years, with the main aim of assessing the determinants of health in the adult population of Porto, Portugal. For the baseline evaluation (1999-2003), 2,485 persons were randomly selected and have been repeatedly evaluated over time. Between October 2006 and July 2008, participants aged 45 years or older were eligible to
Table 1. Clinical and echocardiographic characteristics of the study sample

\begin{tabular}{|c|c|}
\hline Age, years & $61.5 \pm 10.5$ \\
\hline Females & 61.1 \\
\hline BMI & $27.4 \pm 4.6$ \\
\hline Diabetes mellitus & 11.3 \\
\hline Dyslipidemia & 47.8 \\
\hline Hypertension & 54.8 \\
\hline Current smokers & 14.7 \\
\hline Treatment with $\beta$-blockers & 6.6 \\
\hline Heart rate, bpm & $63.4 \pm 9.7$ \\
\hline \multicolumn{2}{|l|}{ Blood pressure, $\mathrm{mm} \mathrm{Hg}$} \\
\hline Systolic & $132.9 \pm 19.6$ \\
\hline Diastolic & $78.3 \pm 11.3$ \\
\hline LA volume, $\mathrm{mL} / \mathrm{m}^{2}$ & $28.5 \pm 9.6$ \\
\hline \multicolumn{2}{|l|}{ LV geometry } \\
\hline Septum, mm & $8.6 \pm 1.5$ \\
\hline Posterior wall, $\mathrm{mm}$ & $7.9 \pm 1.2$ \\
\hline LV mass, $\mathrm{g} / \mathrm{m}^{2}$ & $79.1 \pm 18.3$ \\
\hline $\mathrm{LVEDV}, \mathrm{mL} / \mathrm{m}^{2}$ & $66.0 \pm 16.0$ \\
\hline LVESV, $\mathrm{mL} / \mathrm{m}^{2}$ & $26.6 \pm 8.9$ \\
\hline \multicolumn{2}{|l|}{ Systolic function } \\
\hline Ejection fraction, $\%$ & $60.5 \pm 6.2$ \\
\hline \multicolumn{2}{|l|}{ Diastolic function } \\
\hline Mitral E/A ratio & $0.97 \pm 0.32$ \\
\hline Deceleration time, ms & $235.8 \pm 53.6$ \\
\hline E'velocity, $\mathrm{cm} / \mathrm{s}$ & $10.6 \pm 3.2$ \\
\hline $\mathrm{E} / \mathrm{E}$ ' ratio & $7.3 \pm 2.6$ \\
\hline
\end{tabular}

Values are presented as means \pm SD or percents. The total number of patients is 835. LVEDV, LV end-diastolic volume; LVESV, LV end-systolic volume.

undergo a systematic evaluation of parameters of cardiac structure and function, which included a cardiovascular clinical history, a physical examination, a detailed anthropometric evaluation, collection of fasting blood samples, and a transthoracic echocardiogram. Among the 2,048 cohort members in the eligible age range at that time, 134 (6.5\%) had died, 198 (9.7\%) refused reevaluation, and 580 $(28.3 \%)$ were lost to follow-up. We further excluded 301 patients with a previous myocardial infarction, percutaneous or surgical revascularization, a prior cardiac surgery, significant (moderate to severe) valvular heart disease, a history of thyroid disease, or treatment with drugs that interfere with thyroid function, as well as those without serum available for determination of thyroid function or with $\mathrm{TSH}$, free thyroxine (FT4), or free triiodothyronine (FT3) outside of the reference range $(0.35-4.94 \mu \mathrm{IU} / \mathrm{mL}$ for TSH, $0.70-1.48 \mathrm{ng} / \mathrm{dL}$ for FT4, and $1.71-3.71 \mathrm{pg} / \mathrm{mL}$ for FT3). Eight hundred thirty-five participants were included in our analysis.

\section{Thyroid Function Measurements}

TSH, FT3, and FT4 were measured in serum samples stored at $-80^{\circ} \mathrm{C}$ by an electrochemiluminescence immunoassay using an Abbott Architect i2000 analyzer (Abbott Diagnostics). It has been previously shown that TSH and thyroid hormones can be analyzed reliably in frozen stored samples [22]. 
Table 2. Linear association of thyroid function with heart rate and blood pressure

\begin{tabular}{|c|c|c|c|c|c|c|}
\hline & \multicolumn{2}{|l|}{ TSH $(\mu \mathrm{IU} / \mathrm{mL})$} & \multicolumn{2}{|l|}{ FT4 (ng/dL) } & \multicolumn{2}{|l|}{ FT3 (pg/mL) } \\
\hline \multicolumn{7}{|l|}{ Heart rate (bpm) } \\
\hline Unadjusted & $-0.35(-1.79$ to 1.09$)$ & 0.634 & $2.98(-2.32$ to 8.28$)$ & 0.270 & $1.52(-0.26$ to 3.30$)$ & 0.094 \\
\hline Model $1^{\mathrm{a}}$ & $-0.76(-2.20$ to 0.68$)$ & 0.303 & $2.42(-2.87$ to 7.71$)$ & 0.370 & $1.86(0.08$ to 3.64$)$ & 0.041 \\
\hline Model $2^{b}$ & $-0.75(-2.17$ to 0.67$)$ & 0.299 & $1.38(-3.83$ to 6.60$)$ & 0.603 & $1.99(0.23$ to 3.75$)$ & 0.026 \\
\hline Unadjusted & $0.58(-2.32$ to 3.49$)$ & 0.693 & $7.67(-3.00$ to 18.36$)$ & 0.159 & $-0.15(-3.73$ to 3.43$)$ & 0.935 \\
\hline Model $1^{\mathrm{a}}$ & $0.42(-2.32$ to 3.16$)$ & 0.763 & $1.69(-8.35$ to 11.74$)$ & 0.741 & $2.90(-0.48$ to 6.27$)$ & 0.093 \\
\hline Model $2^{\mathrm{b}}$ & $0.14(-2.15$ to 2.43$)$ & 0.906 & $-1.99(-10.38$ to 6.40$)$ & 0.642 & $1.99(-0.84$ to 4.81$)$ & 0.168 \\
\hline \multicolumn{7}{|l|}{ Diastolic } \\
\hline Unadjusted & $1.77(0.10$ to 3.44$)$ & 0.038 & $1.11(-5.06$ to 7.28$)$ & 0.724 & $1.03(-1.03$ to 3.10$)$ & 0.328 \\
\hline Model $1^{\mathrm{a}}$ & $1.88(0.19$ to 3.57$)$ & 0.029 & $1.62(-4.59$ to 7.83$)$ & 0.609 & $0.81(-1.29$ to 2.90$)$ & 0.450 \\
\hline
\end{tabular}

The values shown were estimated by multiple linear regression, with TSH, FT4, or FT3 as independent variable and heart rate, systolic blood pressure, and diastolic blood pressure as dependent variables, adjusting for several variables as stated. TSH was log-transformed. Participants treated with $\beta$-blockers were excluded from heart rate and blood pressure analyses. Statistically significant associations are shown in bold. ${ }^{a}$ Adjusted for age and sex. ${ }^{b}$ Adjusted for age, sex, BMI, hypertension, diabetes, and current smoking.

Echocardiographic Evaluation and Blood Pressure Evaluation

All echocardiography studies were acquired by 1 of 4 cardiologists, using the same equipment (Hewlett-Packard Sonos 5500). Images were stored for later offline analysis by 2 experienced cardiologists who were blinded to clinical data. Cardiac chamber dimensions and volumes and left ventricular (LV) mass were indexed to body surface area. Diastolic function was assessed by measuring mitral inflow velocities (E-wave, $\mathrm{A}$ wave, and $\mathrm{E} / \mathrm{A}$ ratio) and the E-wave deceleration time and the isovolumetric relaxation time using pulsed-wave Doppler in the apical 4-chamber view. Velocities were recorded at end expiration and averaged over 3 consecutive cardiac cycles. Pulsed-wave tissue Doppler velocities were acquired at end expiration, in the apical 4-chamber view, on the lateral side of the mitral annulus, measuring early diastolic (E') and late diastolic $\left(\mathrm{A}^{\prime}\right)$ velocities and estimating the E/E' ratio accordingly. Systolic function was evaluated by ejection fraction calculation using the modified biplane Simpson rule.

Blood pressure was determined by 2 measurements separated by at least $5 \mathrm{~min}$ after a 10 -min rest. When the difference between measurements was larger than $5 \mathrm{~mm} \mathrm{Hg}$ for systolic or diastolic blood pressure, a third measurement was taken and the mean of the 2 closest values was considered.

\section{Comorbidities and Clinical Definitions}

BMI was calculated as weight in kilograms divided by the square of height in meters. Hypertension was defined as a systolic blood pressure $\geq 140 \mathrm{~mm} \mathrm{Hg}$ or a diastolic blood pressure $\geq 90 \mathrm{~mm}$ $\mathrm{Hg}$ at the time of the visit [23] or as use of antihypertensive drugs. Diabetes was defined as a fasting blood glucose level $\geq 126 \mathrm{mg} / \mathrm{dL}$ [24], a self-reported history of diabetes, or use of diabetes medications. Dyslipidemia was defined as low-density lipoprotein cholesterol $\geq 160 \mathrm{mg} / \mathrm{dL}$, triglycerides $\geq 200 \mathrm{mg} / \mathrm{dL}$, high-density lipoprotein cholesterol $<40 \mathrm{mg} / \mathrm{dL}$ [25], or use of lipid-lowering drugs.

\section{Statistical Analysis}

We used linear regression models to evaluate the associations of TSH, FT4, and FT3 (independent variables) with heart rate, blood pressure, cardiac structure, and cardiac function parameters (dependent variables). These associations were evaluated in unadjusted models and adjusted models for sex and age (model 1) and for sex, age, BMI, diabetes, hypertension, and current smoking (model 2). These variables were included as potential confounders due to their known effects on thyroid function $[26,27]$ and cardiac function [28]. Participants treated with $\beta$-blockers were excluded from analyses of heart rate, blood pressure, and cardiac function. As previous studies have shown some similar cardiac manifestations in participants with hypothyroidism and hyperthyroidism [29], we also assessed nonlinear associations using restricted cubic splines with 3 knots at the 10th, 50th, and 90th percentiles [30]. This approach allows the identification of changes in cardiac structure or function that occur with both increasing and decreasing thyroid function within the normal range.

Continuous variables are presented as means $( \pm S D)$. Categorical variables are presented as percentages. Comparisons between groups were made using $t$ tests and the $\chi^{2}$ test. Two-sided $p<0.05$ was considered statistically significant. Statistical analyses were performed using Stata software, version 14.2 (StataCorp).

\section{Results}

\section{Study Population}

A total of 835 participants (510 women and 325 men) were included in our analysis after applying the inclusion and exclusion criteria. Participants who were ex- 
Table 3. Linear association of thyroid function with cardiac structure



cluded or lost to follow-up did not differ from those included at baseline regarding sex, BMI, or smoking status, but they were older and had a higher prevalence of comorbidities (online suppl. Table 1, see www. karger.com/doi/10.1159/000508407). The general characteristics and echocardiographic findings of the included population are shown in Table 1 . The mean age was 61.5 years (SD 10.5) years. Eleven percent of the participants had diabetes mellitus, nearly half had dyslipidemia $(47.8 \%)$, and more than half had hypertension $(54.8 \%)$.

\section{Association of Thyroid Function with Heart Rate and Blood Pressure}

The associations of thyroid function with heart rate and blood pressure in univariable and multivariable analysis are shown in Table 2. We observed that heart rate increased significantly with increasing FT3 levels after adjustment for age and sex (model 1) and further adjustment for potential confounding factors of BMI, hypertension, diabetes, and smoking (model 2). Regarding blood pressure, mean diastolic blood pressure was positively associated with TSH levels both in univariate and in multivariate regression models.

\section{Association of Thyroid Function with Cardiac \\ Structure}

Concerning cardiac structure (Table 3), both LV enddiastolic and end-systolic volumes were found to be inversely associated with TSH levels in the unadjusted and adjusted regression models. In addition, analysis by restricted cubic splines (Fig. 1) showed U-shaped associations between FT3 levels and LV cavity volumes in unadjusted and adjusted models. 


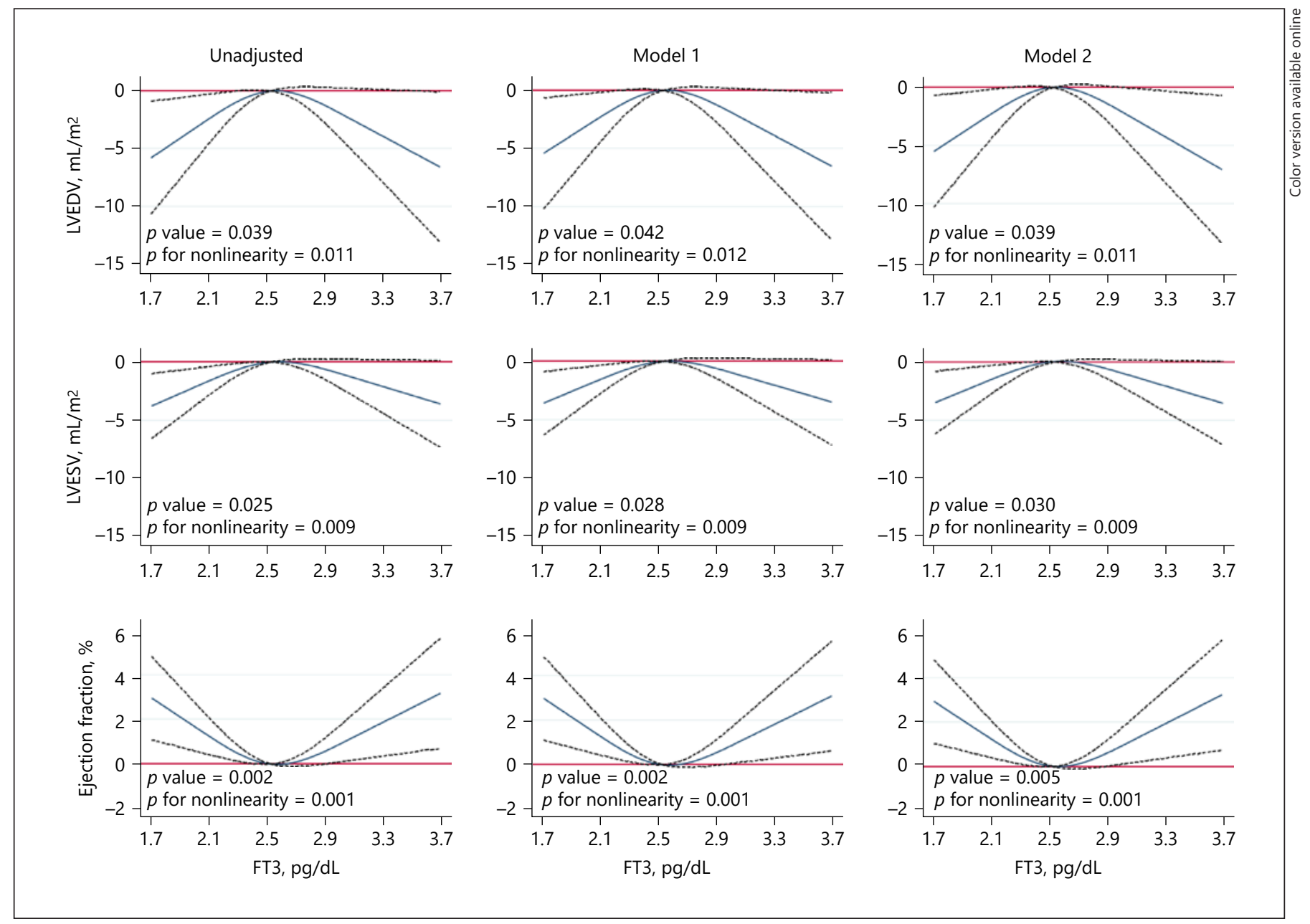

Fig. 1. Restricted cubic splines analysis of the continuous relationship between FT3 and LV end-diastolic volume (LVEDV), LV end-systolic volume (LVESV), and ejection fraction. Participants treated with $\beta$-blockers were excluded from these analyses. Model 1: adjusted for age and sex. Model 2: adjusted for age, sex, BMI, hypertension, diabetes and current smoking.

Negative correlations of FT3 levels with left atrium (LA) volume ( $\beta=-2.44$, range -4.16 to $-0.72 ; p=0.006$ ) and parameters of LV geometry, specifically septum thickness $(\beta=-0.26$, range -0.52 to $-0.003 ; p=0.048)$ and $\mathrm{LV}$ mass $(\beta=-3.57$, range -6.82 to $-0.31 ; p=0.032)$, were also found, but these were not verified after adjustment in models 1 and 2.

Analysis of FT4 levels by restricted cubic splines with septum thickness, posterior wall thickness, and LV mass (Fig. 2) showed only a nonlinear relationship between FT4 and LV posterior wall thickness, with the higher values of posterior wall thickness among participants with higher and lower FT4 levels within the reference range. However, this association was not significant after adjustments in model 2.

\section{Association of Thyroid Function with Systolic and Diastolic Function}

Linear regression analysis between thyroid function and systolic and diastolic functions are shown in Table 4. Concerning systolic function, TSH levels were positively associated with ejection fraction $(\beta=0.96$, range $0.03-1.89 ; p=0.042$ ). This association was no longer significant after adjustments in models 1 and 2. On the other hand, analysis between FT3 levels and ejection fraction by restricted cubic splines (Fig. 1) showed a strong nonlinear association between these variables ( $p$ for nonlinearity $=0.001$ ), with a higher ejection fraction in extreme FT3 levels within the reference range. These associations remained significant after adjustments in both models. 

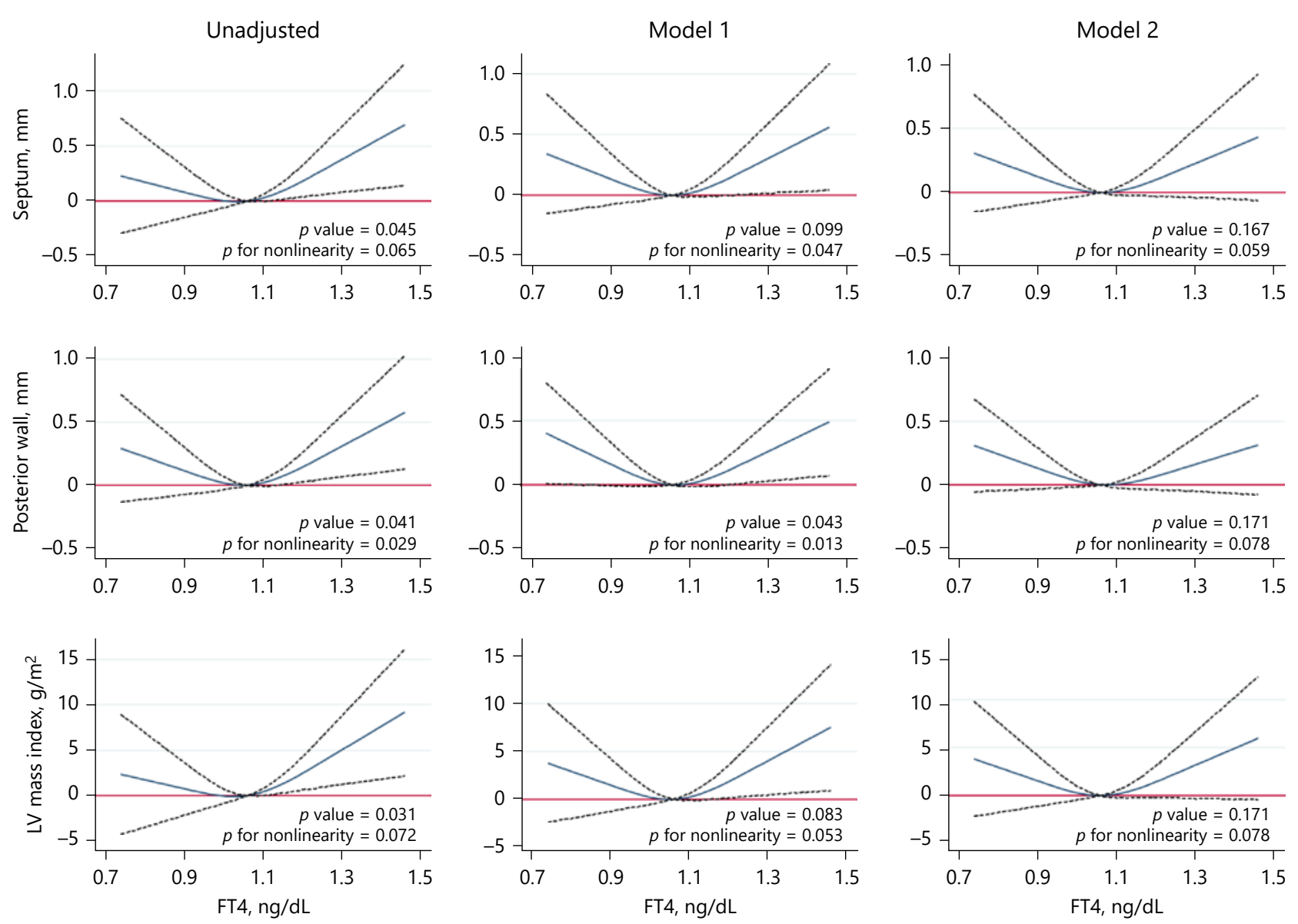

Fig. 2. Restricted cubic splines analysis of the continuous relationship between FT4 and septum thickness, posterior wall thickness, and LV mass. Model 1: adjusted for age and sex. Model 2: adjusted for age, sex, BMI, hypertension, diabetes, and current smoking.

In diastolic function, FT3 levels were positively correlated with E' velocity $(\beta=0.80$; range $0.21-1.39 ; p=0.008)$, unlike FT4 levels, which were negatively correlated with this variable $(\beta=-2.76$, range -4.52 to $-0.99 ; p=0.002)$ as well as with the mitral $\mathrm{E} / \mathrm{A}$ ratio $(\beta=-0.22$, range -0.39 to $-0.04 ; p=0.015)$. Deceleration time increased significantly with increasing FT4 levels $(\beta=29.58$, range $0.43-$ $58.74 ; p=0.047)$. All of these associations were no longer significant after adjustments in models 1 and 2.

\section{Discussion}

In a population-based sample, thyroid hormone levels within the reference range were significantly associated with heart rate, blood pressure, and cardiac structure and function. Our study shows robust positive associations between FT3 levels and heart rate, as well as between TSH levels and diastolic blood pressure, which suggests that increasing thyroid hormone levels are associated with a higher heart rate and a lower diastolic blood pressure. At a structural level, the inverse association found between TSH levels and LV end-systolic and end-diastolic volumes also indicates that an increase in thyroid function is associated with larger LV cavity volumes. On the other hand, nonlinear associations of FT4 and FT3 with LV wall thickness and LV ejection fraction, respectively, suggest that thyroid hormone concentrations near the extremes of the reference range are associated with a greater wall thickness and a higher ejection fraction.

Previous studies evaluating the association of thyroid hormones with cardiac function have shown contradic- 
Table 4. Linear association of thyroid function with systolic and diastolic function

\begin{tabular}{|c|c|c|c|c|c|c|}
\hline & \multicolumn{2}{|l|}{$\mathrm{TSH}(\mu \mathrm{IU} / \mathrm{mL})$} & \multicolumn{2}{|l|}{ FT4 (ng/dL) } & \multicolumn{2}{|l|}{ FT3 (pg/mL) } \\
\hline Unadjusted & $0.96(0.03$ to 1.89$)$ & 0.042 & $-1.42(-4.86$ to 2.01$)$ & 0.416 & $-0.44(-1.59$ to 0.71$)$ & 0.449 \\
\hline Model $1^{\mathrm{a}}$ & $0.78(-0.16$ to 1.71$)$ & 0.103 & $-1.44(-4.87$ to 2.00$)$ & 0.412 & $-0.45(-1.61$ to 0.71$)$ & 0.556 \\
\hline Mitral E/A ratio & & & & & & \\
\hline Unadjusted & $-0.01(-0.04$ to 0.05$)$ & 0.831 & $-0.22(-0.39$ to -0.04$)$ & 0.015 & $0.03(-0.02$ to 0.09$)$ & 0.240 \\
\hline Model $1^{\mathrm{a}}$ & $0.01(-0.03$ to 0.05$)$ & 0.676 & $-0.09(-0.25$ to 0.06$)$ & 0.247 & $-0.03(-0.02$ to 0.02$)$ & 0.293 \\
\hline Model $2^{\mathrm{b}}$ & $0.01(-0.03$ to 0.05$)$ & 0.617 & $-0.08(-0.23$ to 0.07$)$ & 0.296 & $-0.02(-0.07$ to 0.03$)$ & 0.393 \\
\hline \multicolumn{7}{|c|}{ Deceleration time (ms) } \\
\hline Unadjusted & $1.26(-6.63$ to 9.16$)$ & 0.451 & $29.58(0.43$ to 58.74$)$ & 0.047 & $-2.00(-11.75$ to 7.74$)$ & 0.687 \\
\hline Unadjusted & $-0.02(-0.50$ to 0.46$)$ & 0.941 & $-2.76(-4.52$ to -0.99$)$ & 0.002 & $0.80(0.21$ to 1.39$)$ & 0.008 \\
\hline Model $1^{\mathrm{a}}$ & $0.08(-0.33$ to 0.49$)$ & 0.699 & $-1.29(-2.79$ to 0.21$)$ & 0.091 & $0.07(-0.44$ to 0.58$)$ & 0.787 \\
\hline Model $2^{b}$ & $0.09(-0.32$ to 0.49$)$ & 0.674 & $-1.13(-2.62$ to 0.35$)$ & 0.134 & $0.12(-0.38$ to 0.62$)$ & 0.634 \\
\hline \multicolumn{7}{|l|}{ E/E' ratio } \\
\hline Unadjusted & $0.20(-0.17$ to 0.56$)$ & 0.287 & $1.26(-0.08$ to 2.60$)$ & 0.065 & $-0.41(-0.85$ to 0.04$)$ & 0.074 \\
\hline Model $1^{\mathrm{a}}$ & $0.04(-0.29$ to 0.37$)$ & 0.824 & $0.45(-0.77$ to 1.66$)$ & 0.471 & $0.01(-0.40$ to 0.42$)$ & 0.980 \\
\hline Model $2^{\mathrm{b}}$ & $0.04(-0.28$ to 0.37$)$ & 0.789 & $0.23(-0.96$ to 1.42$)$ & 0.701 & $-0.02(-0.42$ to 0.38$)$ & 0.911 \\
\hline
\end{tabular}

tory results. Pearce et al. [31] evaluated the association of TSH with LV structure and function in the Framingham Heart Study. There were no significant associations between TSH and LV mass, LV wall thickness, LA, or LV systolic function. However, TSH $<0.5 \mathrm{mU} / \mathrm{L}$ was associated with improved LV contractility, as assessed by echocardiographic LV fractional shortening, among women, but this parameter is no longer recommended in clinical practice [31]. In that study, associations of $\mathrm{T} 4$ and $\mathrm{T} 3$ with cardiac function were not assessed.

In a study of 318 participants with hypertension (mean age in the euthyroid group: 68 years; $10 \%$ with heart failure), Iida et al. [32] described that thyroid hormones within the normal reference range were positively associated with LV mass index. On the other hand, in a sample restricted to participants aged between 35 and 55 years without hypertension or cardiovascular disease, Roef et al. [33] showed that higher thyroid hormone levels within the normal range were associated with heart rate and smaller LV cavity sizes but not with wall thickness after adjustment for blood pressure. In this study, thyroid hormone levels were positively associated with early and late ventricular filling, in contrast to our findings.

The differences between studies are probably related to the populations evaluated. While the study by Roef et al. [33] included only young and healthy participants, the study by Iida et al. [32] was restricted to older hypertensive individuals. On the other hand, our study represents a particularly important segment of the population. As we evaluated participants aged 45 years or older with a proportion of hypertension, diabetes, and dyslipidemia representative of the general population, our study better represents the population usually targeted for cardiovascular preventive interventions.

Thyroid hormones modulate cardiac function through genomic and nongenomic mechanisms $[8,33,34]$. T3 is the active form of thyroid hormones and it is responsible for most cardiovascular effects. In humans, type 2 deiodinase is expressed in the heart and allows local conversion of T4 to T3 [8]. TSH level is as a sensitive marker of 
thyroid function as TSH secretion increases with decreasing thyroid hormone levels. However, some studies have suggested that TSH may also have direct effects on heart and vessels [35-37]. In the myocardium, thyroid hormone stimulates channels and transporters involved in calcium fluxes, improving both contraction and relaxation. Thyroid hormone also improves myocardial relaxation through upregulation of sarcoplasmic reticulum calcium-activated ATPase (SERCA2) and $\mathrm{Na}^{+} / \mathrm{K}^{+}$ATPase, and downregulation of phospholamban $[8,34]$. On the other hand, thyroid hormones directly induce myocardial hypertrophy through activation of PI3K/ AKT $/ \mathrm{mTOR}$ and GK3 $\beta$ signaling pathways [8]. While the direct effects of thyroid hormones on diastolic function may be more marked in younger healthier individuals (like the population studied by Roef et al. [33]), the hypertrophic effects of thyroid hormones may be more marked in the elderly hypertensive population (like the population studied by Iida et al. [32]). In our population, neither diastolic function nor LV mass was linearly associated with thyroid hormone levels. Interestingly, both subclinical hypo- and hyperthyroidism are associated with diastolic dysfunction and myocardial hypertrophy, probably through different mechanisms $[38,39]$. In our study, FT4 was nonlinearly associated with LV wall thickness. The fact that associations of FT4 with LV wall thickness were no longer significant after adjustment for model 2 suggests that some of the thyroid hormone effects on the LV mass may be mediated by effects on hypertension or the glycemic profile. These nonlinear associations of FT4 with LV wall thickness highlight that the hypertrophic effects of low and high thyroid hormone levels may extend to the normal reference range of thyroid hormones. The absence of associations with diastolic function suggests that the known direct effects of thyroid hormones on cardiac relaxation may be opposed by detrimental effects on myocardial hypertrophy.

The nonlinear association of FT3 with LV ejection fraction is probably the result of 2 different mechanisms. While the increase in LV ejection fraction with increasing FT3 above the median is probably the consequence of the positive inotropic effects of thyroid hormones, the increase in LV ejection fraction with FT3 below the median may be related to the increase in diastolic filling time with decreasing heart rate [40].

Studies in patients with hypo- and hyperthyroidism have shown that heart rate and blood pressure are strongly associated with of thyroid dysfunction [29, 40]. While patients with overt hypothyroidism usually have bradycardia and a high diastolic blood pressure, patients with overt hyperthyroidism usually have tachycardia and increased systolic blood pressure [40, 41]. Our results suggest that, even within the normal range, variations of thyroid function have a relevant effect on heart rate and blood pressure, which has also been suggested by previous studies $[17,42]$. Overt and subclinical hyperthyroidism has also been associated with an increased risk of cardiac arrhythmias [43-45] and with volume overload [46]. In the Rotterdam Study, Chaker et al. $[47,48]$ found that increasing thyroid function was associated with a higher risk of atrial fibrillation in euthyroid participants and a higher risk of sudden cardiac death. The tachycardic effects appear to be dependent on direct cardiac electrophysiological effects [49-51] and $\beta$-adrenergic stimulation [52], while the volume overload is probably mediated by peripheral vasodilation with compensatory activation of the renin-angiotensin-aldosterone axis and increased renal reabsorption of sodium and water [46]. These effects may be observed even within the normal range as suggested by our findings of an increased heart rate with higher FT3 and an association of lower TSH levels with a lower diastolic blood pressure and with higher LV cavity volumes.

Atherosclerotic risk is also thought to be influenced by variations of thyroid hormones within the normal range. Both low and high-normal thyroid functions have been associated with atherosclerotic disease in several population-based cohorts. In the HUNT study, Asvold et al. [53, 54] found that higher TSH levels were associated with a higher risk of coronary heart disease mortality, but only in women. On the other hand, a recent meta-analysis did not find an association between TSH levels and coronary heart disease events and mortality [55]. However, a Ushaped association between FT4 within the normal range and the risk of coronary events was present. A more atherogenic lipid profile with lower FT4 levels may explain the higher cardiovascular risk with a low-normal thyroid status [56]. In another study, Bano et al. [57] showed that a higher FT4 level was associated with a higher risk of subclinical and clinical atherosclerosis. A procoagulant state linked to a high-normal thyroid function may be a possible mechanism to explain this association [58]. Other plausible mechanism would be related to the increasing workload of the heart due to an increased heart rate and myocardial hypertrophy [41].

The results of our analysis are clinically relevant as they highlight that, even within the normal range, thyroid hormones strongly modulate cardiovascular function. The use of detailed echocardiography data in a sample representative of the general population is a strength of our study, as previous studies that evaluated the associa- 
tion of thyroid hormones with echocardiographic parameters focused on specific subpopulations [32, 33]. Furthermore, the identification of a U-shaped association of thyroid hormones with wall thickness and ejection fraction is important as it highlights that both normal-low and normal-high thyroid hormones are associated with a higher ejection fraction and a greater wall thickness. In those treated with levothyroxine the risk of adverse cardiovascular outcomes may increase both with undertreatment and with overtreatment [59]. The clinical impact of small changes in thyroid hormones within the normal range concerning cardiovascular outcomes is still not clear. Although some authors have suggested the use of different targets within the normal range according to the clinical context [60], the evidence for such approach is scarce. Furthermore, treatment with levothyroxine is usually associated with higher T4 and lower T3 levels compared with the general population [46], which may not completely restore the euthyroid state [61]. Further studies, ideally randomized clinical trials, are needed to evaluate the potential cardiovascular benefits of individualized targets and the effects of the combination of T4/T3 treatment in participants treated with thyroid hormones.

Regarding the limitations of our study, the cross-sectional design limits our ability to make causal inferences. We cannot exclude that residual confounding (including drugs and unmeasured comorbidities) or loss to followup of participants initially included in this cohort may have influenced our results. Furthermore, we must highlight that we evaluated the association of thyroid function within the normal range with cardiac function at a population level. Thyroid function has an important interindividual variation that may have decreased our ability to detect significant associations [62]. Prospective studies are needed to evaluate the effects of intraindividual variations of thyroid hormone levels. As we only evaluated participants who were 45 years or older, our results may not be applicable to younger individuals. Regarding the assessment of echocardiography data, we did not evaluate intraobserver or interobserver variability. However, all of the cardiologists involved in this study had extensive experience in echocardiography and worked at the same institution, and a detailed procedure protocol was discussed between the team, prior to the start of the study, to harmonize the methodology and the measurements. Finally, we only evaluated free thyroid hormone concentrations. Although only the free fraction is considered biologically active $[63,64]$, we cannot exclude that different associations could have been found if we had evaluated total thyroid hormone concentrations.
In conclusion, variations of thyroid function within the reference range are associated with heart rate, blood pressure, cardiac structure, and cardiac function in the general population. Our results suggest that increasing thyroid function (lower TSH, higher FT4, or higher FT3) is associated with a higher heart rate, a lower diastolic blood pressure, and larger LV cavity volumes. On the other hand, both LV wall thickness and LV ejection fraction have a U-shaped association with thyroid hormones, with a greater wall thickness and a higher ejection fraction near the extremes of the normal range of thyroid hormone concentrations.

\section{Statement of Ethics}

Consent was obtained from each subject after a full explanation of the purpose and nature of all of the procedures used. This study was approved by the Ethics Committee of the São João Hospital Center/Faculty of Medicine of University of Porto.

\section{Conflict of Interest Statement}

The authors have no conflict of interests to declare.

\section{Funding Sources}

This work was supported by the DOCnet project (NORTE01-0145-FEDER-000003), supported by Norte Portugal Regional Operational Program (NORTE 2020), under the PORTUGAL 2020 Partnership Agreement, through the European Regional Development Fund (ERDF), and the NETDIAMOND project (POCI-01-0145-FEDER-016385), supported by European Structural and Investment Funds, Lisbon's Regional Operational Program 2020, and national funds from the Portuguese Foundation for Science and Technology - both projects through the Cardiovascular Research Center (UnIC, FCT 51/94) - and by the Portuguese Foundation for Science and Technology (grant POCI/ SAU-ESP/61492/2004) and the Unidade de Investigação em Epidemiologia - Instituto de Saúde Pública da Universidade do Porto (EPIUnit) (POCI-01-0145-FEDER-006862, ref. UID/ DTP/04750/2013).

\section{Author Contributions}

J.S.N., R.F.-C., M.B.-C., A.R.L., and A.A. contributed to the conception and design of this work, the statistical analysis, and interpretation of data. S.M., A.O., J.T.G., D.C., and A.L.-M. contributed to interpretation of data. J.S.N., M.B.-C., and A.R.L. prepared the first draft of this paper. All of the authors critically revised this work and approved the final version of this paper. 


\section{References}

1 Yusuf S, Hawken S, Ounpuu S, Dans T, Avezum A, Lanas F, et al.; INTERHEART Study Investigators. Effect of potentially modifiable risk factors associated with myocardial infarction in 52 countries (the INTERHEART study): case-control study. Lancet. 2004 Sep; 364(9438):937-52.

2 Gaede P, Lund-Andersen H, Parving HH, Pedersen O. Effect of a multifactorial intervention on mortality in type 2 diabetes. $\mathrm{N}$ Engl J Med. 2008 Feb;358(6):580-91.

3 Baigent C, Blackwell L, Emberson J, Holland LE, Reith C, Bhala N, et al.; Cholesterol Treatment Trialists' (CTT) Collaboration. Efficacy and safety of more intensive lowering of LDL cholesterol: a meta-analysis of data from 170,000 participants in 26 randomised trials. Lancet. 2010 Nov;376(9753):1670-81.

4 Wright JT Jr, Williamson JD, Whelton PK, Snyder JK, Sink KM, Rocco MV, et al.; SPRINT Research Group. A Randomized Trial of Intensive versus Standard Blood-Pressure Control. N Engl J Med. 2015 Nov; 373(22):2103-16.

5 Lieb W, Enserro DM, Sullivan LM, Vasan RS. Residual Cardiovascular Risk in Individuals on Blood Pressure-Lowering Treatment. J Am Heart Assoc. 2015 Nov;4(11):e002155.

6 Dash S, Leiter LA. Residual cardiovascular risk among people with diabetes. Diabetes Obes Metab. 2019 Apr;21(Suppl 1):28-38.

7 Cooper DS, Biondi B. Subclinical thyroid disease. Lancet. 2012 Mar;379(9821):1142-54.

8 Jabbar A, Pingitore A, Pearce SH, Zaman A, Iervasi G, Razvi S. Thyroid hormones and cardiovascular disease. Nat Rev Cardiol. 2017 Jan;14(1):39-55.

9 Chaker L, Bianco AC, Jonklaas J, Peeters RP. Hypothyroidism. Lancet. 2017 Sep;390 (10101):1550-62

10 De Leo S, Lee SY, Braverman LE. Hyperthyroidism. Lancet. 2016 Aug;388(10047):90618.

11 Hak AE, Pols HA, Visser TJ, Drexhage HA, Hofman A, Witteman JC. Subclinical hypothyroidism is an independent risk factor for atherosclerosis and myocardial infarction in elderly women: the Rotterdam Study. Ann Intern Med. 2000 Feb;132(4):270-8.

12 Selmer C, Olesen JB, Hansen ML, von Kappelgaard LM, Madsen JC, Hansen PR, et al. Subclinical and overt thyroid dysfunction and risk of all-cause mortality and cardiovascular events: a large population study. J Clin Endocrinol Metab. 2014 Jul;99(7):2372-82.

13 Gencer B, Collet TH, Virgini V, Bauer DC, Gussekloo J, Cappola AR, et al.; Thyroid Studies Collaboration. Subclinical thyroid dysfunction and the risk of heart failure events: an individual participant data analysis from 6 prospective cohorts. Circulation. 2012 Aug; 126(9):1040-9.

14 Rodondi N, den Elzen WP, Bauer DC, Cappola AR, Razvi S, Walsh JP, et al.; Thyroid Studies Collaboration. Subclinical hypothy- roidism and the risk of coronary heart disease and mortality. JAMA. 2010 Sep;304(12): 1365-74.

15 Collet TH, Gussekloo J, Bauer DC, den Elzen WP, Cappola AR, Balmer P, et al.; Thyroid Studies Collaboration. Subclinical hyperthyroidism and the risk of coronary heart disease and mortality. Arch Intern Med. 2012 May; 172(10):799-809.

16 Sawin CT, Geller A, Wolf PA, Belanger AJ, Baker E, Bacharach P, et al. Low serum thyrotropin concentrations as a risk factor for atrial fibrillation in older persons. N Engl J Med. 1994 Nov;331(19):1249-52.

17 Asvold BO, Bjøro T, Nilsen TI, Vatten LJ. Association between blood pressure and serum thyroid-stimulating hormone concentration within the reference range: a populationbased study. J Clin Endocrinol Metab. 2007 Mar;92(3):841-5.

18 Ittermann $\mathrm{T}$, Thamm $\mathrm{M}$, Wallaschofski $\mathrm{H}$, Rettig R, Völzke H. Serum thyroid-stimulating hormone levels are associated with blood pressure in children and adolescents. J Clin Endocrinol Metab. 2012 Mar;97(3):828-34.

19 Vale C, Neves JS, von Hafe M, Borges-Canha M, Leite-Moreira A. The Role of Thyroid Hormones in Heart Failure. Cardiovasc Drugs Ther. 2019 Apr;33(2):179-88.

20 von Hafe M, Neves JS, Vale C, Borges-Canha M, Leite-Moreira A. The impact of thyroid hormone dysfunction on ischemic heart disease. Endocr Connect. 2019 May;8(5):R7690.

21 Santos AC, Ebrahim S, Barros H. Alcohol intake, smoking, sleeping hours, physical activity and the metabolic syndrome. Prev Med. 2007 Apr;44(4):328-34.

22 Männistö T, Surcel HM, Bloigu A, Ruokonen A, Hartikainen AL, Järvelin MR, et al. The effect of freezing, thawing, and short- and longterm storage on serum thyrotropin, thyroid hormones, and thyroid autoantibodies: implications for analyzing samples stored in serum banks. Clin Chem. 2007 Nov;53(11): 1986-7.

23 Williams B, Mancia G, Spiering W, Agabiti Rosei E, Azizi M, Burnier M, et al.; ESC Scientific Document Group. 2018 ESC/ESH Guidelines for the management of arterial hypertension. Eur Heart J. 2018 Sep;39(33):3021104.

24 American Diabetes Association. 2. Classification and Diagnosis of Diabetes: Standards of Medical Care in Diabetes-2019. Diabetes Care. 2019 Jan;42 Suppl 1:S13-28.

25 Expert Panel on Detection, Evaluation, and Treatment of High Blood Cholesterol in Adults. Executive Summary of The Third Report of The National Cholesterol Education Program (NCEP) Expert Panel on Detection, Evaluation, And Treatment of High Blood Cholesterol In Adults (Adult Treatment Panel III). JAMA. 2001 May;285(19): 2486-97.
26 Reinehr T. Obesity and thyroid function. Mol Cell Endocrinol. 2010 Mar;316(2):165-71.

27 Laclaustra M, Moreno-Franco B, LouBonafonte JM, Mateo-Gallego R, Casasnovas JA, Guallar-Castillon P, et al. Impaired Sensitivity to Thyroid Hormones Is Associated With Diabetes and Metabolic Syndrome. Diabetes Care. 2019 Feb;42(2):303-10.

28 Ziaeian B, Fonarow GC. Epidemiology and aetiology of heart failure. Nat Rev Cardiol. 2016 Jun;13(6):368-78.

29 Razvi S, Jabbar A, Pingitore A, Danzi S, Biondi B, Klein I, et al. Thyroid Hormones and Cardiovascular Function and Diseases. J Am Coll Cardiol. 2018 Apr;71(16):1781-96.

30 Harrell FE Jr, Lee KL, Pollock BG. Regression models in clinical studies: determining relationships between predictors and response. J Natl Cancer Inst. 1988 Oct;80(15):1198-202.

31 Pearce EN, Yang Q, Benjamin EJ, Aragam J, Vasan RS. Thyroid function and left ventricular structure and function in the Framingham Heart Study. Thyroid. 2010 Apr;20(4):36973.

32 Iida M, Yamamoto M, Ishiguro Y, Yamazaki M, Honjo H, Kamiya K. Thyroid hormone within the normal range is associated with left ventricular mass in patients with hypertension. J Am Soc Hypertens. 2012 Jul-Aug;6(4): 261-9.

33 Roef GL, Taes YE, Kaufman JM, Van Daele CM, De Buyzere ML, Gillebert TC, et al. Thyroid hormone levels within reference range are associated with heart rate, cardiac structure, and function in middle-aged men and women. Thyroid. 2013 Aug;23(8):947-54.

34 Vargas-Uricoechea H, Sierra-Torres CH. Thyroid hormones and the heart. Horm Mol Biol Clin Investig. 2014 Apr;18(1):15-26.

35 Dong J, Gao C, Liu J, Cao Y, Tian L. TSH inhibits SERCA2a and the PKA/PLN pathway in rat cardiomyocytes. Oncotarget. 2016 Jun; 7(26):39207-15.

36 Fernandez-Ruocco J, Gallego M, Rodriguezde-Yurre A, Zayas-Arrabal J, Echeazarra L, Alquiza A, et al. High Thyrotropin Is Critical for Cardiac Electrical Remodeling and Arrhythmia Vulnerability in Hypothyroidism. Thyroid. 2019 Jul;29(7):934-45.

37 Tian L, Ni J, Guo T, Liu J, Dang Y, Guo Q, et al. TSH stimulates the proliferation of vascular smooth muscle cells. Endocrine. 2014 Aug; 46(3):651-8

38 Monzani F, Di Bello V, Caraccio N, Bertini A, Giorgi D, Giusti C, et al. Effect of levothyroxine on cardiac function and structure in subclinical hypothyroidism: a double blind, placebo-controlled study. J Clin Endocrinol Metab. 2001 Mar;86(3):1110-5.

39 Yue WS, Chong BH, Zhang XH, Liao SY, Jim $\mathrm{MH}$, Kung AW, et al. Hyperthyroidism-induced left ventricular diastolic dysfunction: implication in hyperthyroidism-related heart failure. Clin Endocrinol (Oxf). 2011 May; 74(5):636-43. 
40 Biondi B, Palmieri EA, Lombardi G, Fazio S. Effects of thyroid hormone on cardiac function: the relative importance of heart rate, loading conditions, and myocardial contractility in the regulation of cardiac performance in human hyperthyroidism. J Clin Endocrinol Metab. 2002 Mar;87(3):968-74.

41 Klein I, Danzi S. Thyroid disease and the heart. Circulation. 2007 Oct;116(15):172535.

42 Asvold BO, Bjøro T, Vatten LJ. Associations of TSH levels within the reference range with future blood pressure and lipid concentrations: 11-year follow-up of the HUNT study. Eur J Endocrinol. 2013 Jun;169(1):73-82.

43 Frost L, Vestergaard P, Mosekilde L. Hyperthyroidism and risk of atrial fibrillation or flutter: a population-based study. Arch Intern Med. 2004 Aug;164(15):1675-8.

44 Sgarbi JA, Villaça FG, Garbeline B, Villar HE, Romaldini JH. The effects of early antithyroid therapy for endogenous subclinical hyperthyroidism in clinical and heart abnormalities. J Clin Endocrinol Metab. 2003 Apr;88(4): $1672-7$.

45 Kaminski G, Dziuk M, Szczepanek-Parulska E, Zybek-Kocik A, Ruchala M. Electrocardiographic and scintigraphic evaluation of patients with subclinical hyperthyroidism during workout. Endocrine. 2016 Aug;53(2): $512-9$.

46 Biondi B. Mechanisms in endocrinology: heart failure and thyroid dysfunction. Eur J Endocrinol. 2012 Nov;167(5):609-18.

47 Chaker L, Heeringa J, Dehghan A, Medici M, Visser WE, Baumgartner C, et al. Normal Thyroid Function and the Risk of Atrial Fibrillation: the Rotterdam Study. J Clin Endocrinol Metab. 2015 Oct;100(10):3718-24.

48 Chaker L, van den Berg ME, Niemeijer MN, Franco OH, Dehghan A, Hofman A, et al.
Thyroid Function and Sudden Cardiac Death: A Prospective Population-Based Cohort Study. Circulation. 2016 Sep;134(10):713-22.

49 Renaudon B, Lenfant J, Decressac S, Bois P. Thyroid hormone increases the conductance density of f-channels in rabbit sino-atrial node cells. Receptors Channels. 2000;7(1):1-8.

50 Sun ZQ, Ojamaa K, Nakamura TY, Artman M, Klein I, Coetzee WA. Thyroid hormone increases pacemaker activity in rat neonatal atrial myocytes. J Mol Cell Cardiol. 2001 Apr; 33(4):811-24.

51 Sun ZQ, Ojamaa K, Coetzee WA, Artman M, Klein I. Effects of thyroid hormone on action potential and repolarizing currents in rat ventricular myocytes. Am J Physiol Endocrinol Metab. 2000 Feb;278(2):E302-7.

52 Silva JE, Bianco SD. Thyroid-adrenergic interactions: physiological and clinical implications. Thyroid. 2008 Feb;18(2):157-65.

53 Asvold BO, Bjøro T, Nilsen TI, Gunnell D, Vatten LJ. Thyrotropin levels and risk of fatal coronary heart disease: the HUNT study. Arch Intern Med. 2008 Apr;168(8):855-60.

54 Asvold BO, Bjøro T, Platou C, Vatten LJ. Thyroid function and the risk of coronary heart disease: 12-year follow-up of the HUNT study in Norway. Clin Endocrinol (Oxf). 2012 Dec; 77(6):911-7.

55 Åsvold BO, Vatten LJ, Bjøro T, Bauer DC, Bremner A, Cappola AR, et al.; Thyroid Studies Collaboration. Thyroid function within the normal range and risk of coronary heart disease: an individual participant data analysis of 14 cohorts. JAMA Intern Med. 2015 Jun; 175(6):1037-47.

56 Roos A, Bakker SJ, Links TP, Gans RO, Wolffenbuttel $\mathrm{BH}$. Thyroid function is associated with components of the metabolic syndrome in euthyroid subjects. J Clin Endocrinol Metab. 2007 Feb;92(2):491-6.
57 Bano A, Chaker L, Mattace-Raso FU, van der Lugt A, Ikram MA, Franco OH, et al. Thyroid Function and the Risk of Atherosclerotic Cardiovascular Morbidity and Mortality: the Rotterdam Study. Circ Res. 2017 Dec;121(12): 1392-400.

58 Bano A, Chaker L, de Maat MP, Atiq F, Kavousi $\mathrm{M}$, Franco $\mathrm{OH}$, et al. Thyroid Function and Cardiovascular Disease: The Mediating Role of Coagulation Factors. J Clin Endocrinol Metab. 2019 Aug;104(8):3203-12.

59 Thayakaran R, Adderley NJ, Sainsbury C, Torlinska B, Boelaert K, Šumilo D, et al. Thyroid replacement therapy, thyroid stimulating hormone concentrations, and long term health outcomes in patients with hypothyroidism: longitudinal study. BMJ. 2019 Sep; 366:14892.

60 Hoermann R, Midgley JE, Larisch R, Dietrich JW. Homeostatic Control of the Thyroid-Pituitary Axis: Perspectives for Diagnosis and Treatment. Front Endocrinol (Lausanne). 2015 Nov; 6:177.

61 Peterson SJ, McAninch EA, Bianco AC. Is a Normal TSH Synonymous With "Euthyroidism" in Levothyroxine Monotherapy? J Clin Endocrinol Metab. 2016 Dec;101(12):496473.

62 Andersen S, Pedersen KM, Bruun NH, Laurberg P. Narrow individual variations in serum $\mathrm{T}(4)$ and $\mathrm{T}(3)$ in normal subjects: a clue to the understanding of subclinical thyroid disease. J Clin Endocrinol Metab. 2002 Mar;87(3): 1068-72.

63 Faix JD. Principles and pitfalls of free hormone measurements. Best Pract Res Clin Endocrinol Metab. 2013 Oct;27(5):631-45.

64 Thienpont LM, Van Uytfanghe K, Poppe K, Velkeniers B. Determination of free thyroid hormones. Best Pract Res Clin Endocrinol Metab. 2013 Oct;27(5):689-700. 